\title{
Mannschaftsarzt beim FC Basel
}

\section{Tanja Kühnle}

Dipl.-Biol., Managing Editor

Die letzte Prüfung ist geschafft, das Medizinstudium beendet. Und wie geht es jetzt weiter? In unserer Serie «Du findest Deinen Weg!» stellen wir Ihnen in unregelmässigen Abständen aussergewöhnliche Berufsperspektiven für Mediziner vor. In dieser Ausgabe berichtet Dr. med. Markus Rothweiler von seiner Tätigkeit als Mannschaftsarzt beim FC Basel 1893.

Herr Rothweiler, wie kam es, dass Sie Mannschaftsarzt beim FC Basel 1893 geworden sind?

Nach Absolvierung des Diplomkurses für Sportmedizin 1987 und 1988 bekam mein damaliger Chef im Kantonsspital Bruderholz, Professor Dr. med. Werner Müller, mit, dass ich mich für Sport und den FC Basel interessiere. Er selbst war über viele Jahre lang der betreuende Orthopäde des FC Basel und schlug vor, für Ihn in der Klinik die Frontarbeit für den Club zu übernehmen. Daraufhin vertrat ich immer wieder mal den damaligen Mannschaftsarzt Dr. med. Felix Marti bei diversen Spielen und teilweise im Trainingslager. 1996 gründete ich meine eigene Praxis, wodurch sich eine Pause in der Betreuung des FC Basel ergab. 2002 wurde mein Kollege und Praxiscompagnon Dr. med. Markus Weber gebeten, ein Konzept für eine medizinische Abteilung beim FC Basel zu erarbeiten. Er fragte mich dann sofort, ob ich mitmachen würde. Wir hatten schon früher zusammen für den Club gearbeitet und es war klar, dass das eine Person allein neben der Praxisarbeit nicht leisten kann. So wurden wir vom damaligen Präsidenten Rene C. Jäggi als Clubärzte berufen und machen das nun seit 2002.

\section{Sie sind FC-Basel-Fan?}

Ja, ich schaute schon mit meinem Vater jedes Wochenende Fussball im Sportprogramm an. Wir waren immer wieder im Stadion in Basel. Auch mit Schulkollegen fuhr ich mit der Bahn zum Cup-Finale und besuchte in jener Zeit die Meisterfeiern auf dem Marktplatz.

\section{Über den FC Basel 1893}

Der FC Basel wurde 1893 gegründet und spielt in der Raiffeisen Super League, der höchsten Schweizer Fussballliga. Der Verein ist bereits 19 Mal Schweizer Meister geworden und hält diesen Titel aktuell seit 7 Jahren in Folge. Auch international ist der FC Basel präsent: So erreichte er zuletzt 2013 das Europa-League-Halbfinale und 2015 das Achtelfinale in der Champions League. Das St. Jakob-Stadion wurde 2001 neu gebaut und fasst insgesamt 38512 Zuschauer. Mehr Informationen unter www.fcb.ch

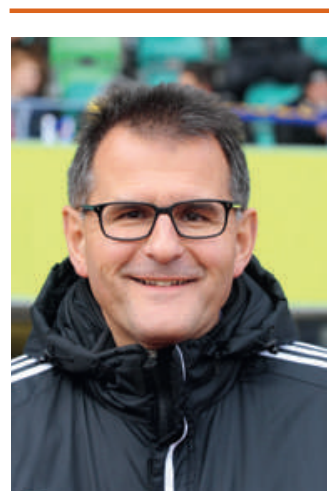

Name: Dr. Markus Rothweiler Alter: 59

Zivilstand: verheiratet, vier Kinder Beim FC Basel tätig seit: 2002 (von 1988-96 bereits in Stellvertretung an der Betreuung beteiligt) Funktion: einer von drei Mannschaftsärzten Ausbildung: Facharzt für orthopädische Chirurgie, Fähigkeitsausweis für Sportmedizin

\section{Und spielen Sie auch selbst Fussball?}

Ich spiele selbst gerne Fussball, war aber nie im Verein.

\section{Gibt es spezielle Voraussetzungen für die Tätigkeit als Mannschaftsarzt?}

Man sollte von der Sportmedizin nicht nur eine kleine Ahnung, sondern, wenn es geht, Sportmedizin und Sporttraumatologie im Curriculum haben. Zudem sollte man den betreffenden Sport mögen. Und man muss sich bewusst sein, dass die Betreuung von Sportlern nach anderen Kriterien läuft, denn hier spielt der Zeitfaktor eine ganz wesentliche Rolle:

Wenn sich ein Spieler verletzt, möchte er sofort behandelt werden und nicht warten, bis irgendwann irgendjemand Zeit hat. Auch der Trainer möchte schnell wissen, ob der Spieler einsatzbereit ist und beispielsweise am nächsten Tag mit zu einem Auslandspiel reisen kann. Da kommt es immer wieder vor, dass die Abklärung an einem Sonntag erfolgt.

\section{Wie viele Ärzte sind für den FC Basel tätig?}

Wir sind ein Dreierteam: Dr. med. Felix Marti, Allgemeinmediziner und Internist, Dr. med. Markus Weber, Rheumatologe und Sportmediziner, und ich, Orthopäde und ebenfalls Sportmediziner. 


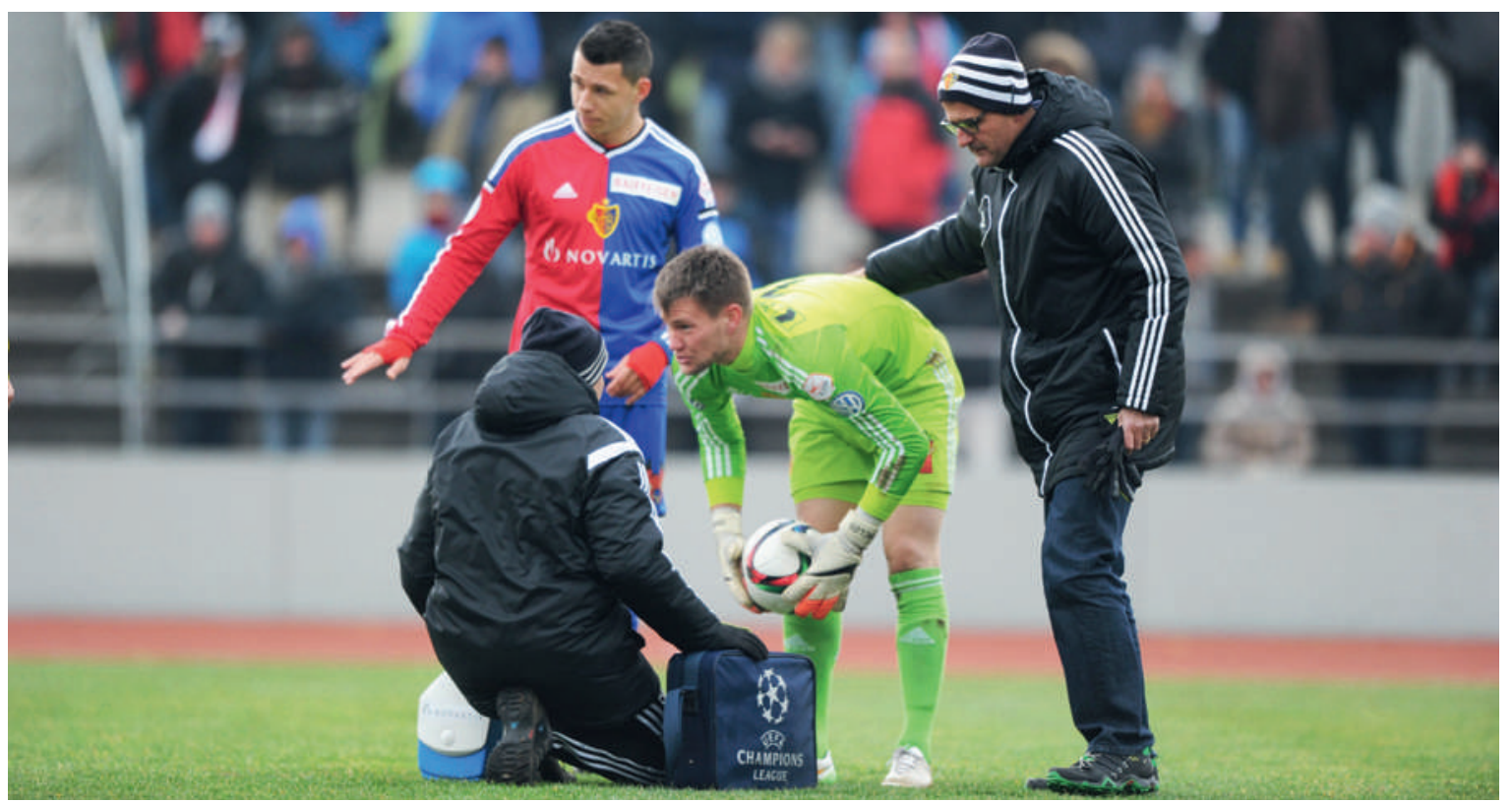

Markus Rothweiler im Einsatz: Der Torwart des FC Basel 1893 benötigt während eines Spiels seine Hilfe.

Jeder von uns ist vollberuflich in einer Praxis tätig. Ich bin dazu auch operativ tätig.

\section{Sind Sie jeweils für bestimmte Spieler zuständig?}

Nein. Sobald der neue Spielplan herauskommt, besprechen wir, wer welches Wochenende und welche Spiele übernimmt. Ob das Spiel auf einen Samstag oder Sonntag fällt, wissen wir aber meist erst einige Wochen vorher. Mit den Vorbereitungs- und Trainingsspielen haben wir 70 bis 75 Spiele im Jahr, die teilen wir uns zu je einem Drittel auf. Auch bei den Trainingslagern ist immer einer von uns Ärzten dabei. Bei Auslandsspielen, die entweder dienstags oder mittwochs stattfinden, müssen wir wegen der An- und Abreise immer insgesamt 4 Tage einplanen. Erst nach der Auslosung wissen wir, ob wir auswärts oder zu Hause spielen und ob der Termin auf einen Dienstag oder einen Mittwoch fällt.

\section{Und wer ist im Notfall Ansprechpartner?}

Im Notfall rufen die Physiotherapeuten des FC Basel einen von uns Ärzten an. Derjenige, den sie zuerst erreichen, behandelt den Spieler dann entweder selbst oder er koordiniert mit den Kollegen, wer übernehmen kann.

\section{Operieren Sie die Spieler, falls nötig?}

Ich habe auch schon Spieler selbst operiert, aber meist arbeiten wir seit vielen Jahren mit Kollegen zusammen, mit denen ich gemeinsam im Team bei Professor Werner Müller, mein Chef während meiner Ausbildungszeit, tätig war. Professor Werner Müller war damals der Operateur des FC Basel.
Wer ist zuständig, wenn sich ein Spieler bei einem Nationalmannschaftsspiel verletzt?

Die Nationalmannschaft hat ihre eigenen Ärzte, mit denen wir in sehr gutem Kontakt stehen. Wenn beispielsweise vor der EM gewisse Pflichtuntersuchungen auf Weisung der UEFA oder FIFA durchgeführt werden müssen, finden diese für Spieler des FC Basel bei uns oder durch uns organisiert statt. Die Resultate liefern wir dann den Nationalmannschaftsärzten. Umgekehrt: Wenn sich einer unserer Spieler verletzt, dann nimmt der Nationalmannschaftsarzt mit uns Kontakt auf und wir besprechen das weitere Vorgehen.

\section{Wie sieht ein typischer Arbeitstag aus?}

Von Montag bis Freitag bin ich für gewöhnlich in der Praxis und in der Klinik. Um 7 Uhr ist Visite, ab 8 Uhr dann Sprechstunde oder OP-Tätigkeit. Abends erledige ich administrative Aufgaben.

Dazu kommt dann noch die Arbeit für den FC Basel: Wenn sich ein Spieler verletzt hat, dann kommt er sofort in die Praxis. Und für die Wochentagsspiele und Trainingslager sperren wir dann unsere Praxistätigkeit.

\section{Können Sie sich an eine besonders schöne Auslands- reise mit dem FC Basel erinnern?}

Von sportlicher Seite her hatten wir ein paar ganz tolle Spiele in England, die uns teils unerwartet weitergebracht haben. Zudem ist in England die Stadionathmosphäre grandios! Manchester, London, Liverpool - das waren sehr beeindruckende Stationen.

Historisch gesehen war eine Reise nach Mostar sehr eindrücklich. Die Stadt war im Balkankrieg von zwei 


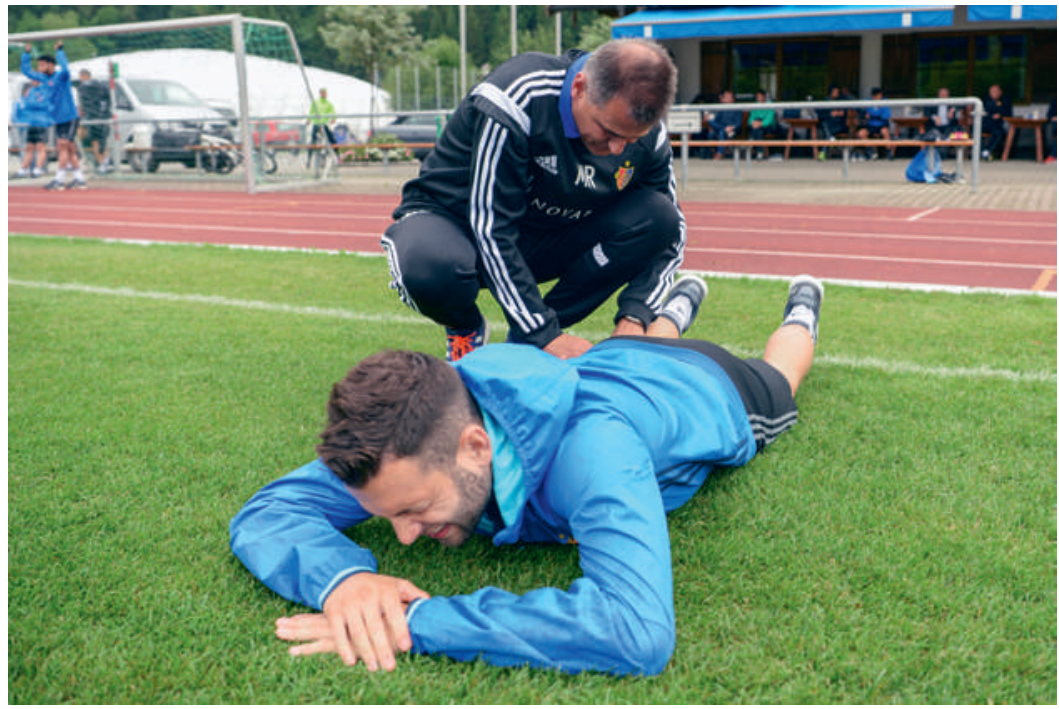

Untersuchung eines Spielers während des Trainingslagers nach dem Zusammenprall mit einem Mitspieler.

\section{Muss Ihnen der Schiedsrichter immer erlauben, das Spielfeld zu betreten?}

Ja, normalerweise muss uns der Schiedsrichter ein Zeichen geben, dass wir aufs Feld rennen dürfen. Aber es gab auch die Situation, dass wir sahen, wie ein Spieler reglos liegen blieb, weil sein Kopf bei einem Zusammenstoss beteiligt war. Da sind wir dann einfach losgerannt. Und das gibt dann auch kein Problem mit dem Schiedsrichter.

\section{Was fasziniert Sie am meisten an Ihrem Beruf?}

Dass mein Beruf so abwechslungsreich ist. Ich habe eine eigene Praxis mit der Sprechstunde, ich operiere und ich betreue die Sportler - das gefällt mir.

\section{Lässt sich Ihr Beruf gut mit der Familie vereinbaren?} Wenn man eine Familie, insbesondere eine Ehefrau hat, die weiss, auf was sie sich einlässt, wenn sie mit einem Arzt verheiratet ist, der in seiner Freizeit zusätzlich seiner sportmedizinischen Tätigkeit nachgeht, dann lässt sich das vereinbaren. Aber man muss einen ganz starken Rückhalt haben in der Familie. Und es muss Verständnis dafür da sein, dass man unter Umständen ein ganzes Wochenende lang im Einsatz ist.

\section{Ist Ihre Frau auch manchmal mit im Stadion?}

Ja, wir gehen ab und zu mal auf unsere Tribünenplätze und geniessen ein Spiel als Zuschauer. Meine Frau ist auch regelmässig mit einem unserer Kinder im Stadion, wenn ich Einsatz habe. Auch auf Auslandsreisen zu internationalen Spielen konnte meine Frau gelegentlich mitkommen, allerdings in der Reisegruppe und nicht im Umfeld des Teams.

\section{Ihr Ratschlag für junge Kollegen, die auch Mann- schaftsarzt werden möchten?}

Primär ist sicher eine fundierte Basis in der Sportmedizin und Sporttraumatologie wichtig. Die Sportmedizin stellt hohe Ansprüche und denen muss man gerecht werden. Zweitens: Man sollte diese Tätigkeit nicht wählen, um sich zu profilieren. Medienauftritte könnten verlockend sein. Aber alle Informationen, die wir herausgeben, werden nach Absprache über unsere Medienabteilung kommuniziert. Der Sportarzt zeichnet sich durch seriöse Arbeit im Hintergrund aus.

Bildnachweise (C) FC Basel 1893 